\title{
Semiconductor Diode Laser provides Desirable Smile: A Clinical and Histological Study with 1-year Follow-up
}

\author{
Abhishek Gautam
}

\begin{abstract}
Aim: The semiconductor diode (SCD) laser is a compact, costeffective, and versatile tool for performing soft tissues applications. The advantage of laser includes a relatively bloodless operating field, sterilization of the wound site, minimal swelling and scaring, reduction of surgical time, and less postoperative pain to the patients in dentistry. This technique is successfully used to evaluate the effects for removal of gingival melanin hyperpigmentation and to assess the color of gingiva pain and wound healing, along with the appearance of gingival repigmentation by clinical and histological examination, in the present study.
\end{abstract}

Materials and methods: For this purpose, five patients were studied which includes four females and one male, aged between 19 and 40, where four patients were nonsmokers and one was a smoker and found periodontally healthy and had no systemic diseases.

Results: The SCD laser found effective in removing gingival melanin pigmentation, and no bleeding with any significant pain was reported by patients. However, repigmentations were observed with Fontana-Masson staining.

Conclusion: These result pointed out that SCD laser is good and safe for removal of pigmented gingiva without local anesthesia. The postoperative period is comfortable for the patient, and healing is fast and good.

Clinical significance: Semiconductor diode laser is an easy and effective tool for removal of gingiva hyperpigmentation. Ablation of gingival hyperpigmented areas was accomplished without any bleeding complication or slight pain, and no pain was observed which provide clean field during time of procedures, and there was uneventful healing without any complication. No recurrence or slight recurrence of pigmentation had been found in 12-month follow-up.

Keywords: Depigmentation, Hyperpigmentation, Repigmentation, Semiconductor diode laser.

How to cite this article: Gautam A. Semiconductor Diode Laser provides Desirable Smile: A Clinical and Histological Study with 1-year Follow-up. Int J Laser Dent 2015;5(2):38-42.

Source of support: Nil

Conflict of interest: None

Practitioner

Department of Periodontology, Chandra Dental College and Hospital, Barabanki, Uttar Pradesh, India

Corresponding Author: Abhishek Gautam, Practitioner Department of Periodontology, Chandra Dental College and Hospital, Barabanki, Uttar Pradesh, India, Phone: +919506305424 e-mail: dr.abhi90@gmail.com

\section{INTRODUCTION}

Specific targeting of melanosomes may allow for laser therapy of pigmented cutaneous lesion in dermatology and plastic surgery. ${ }^{1-3}$ In dentistry, melanin pigmentation of the gingiva is considered to be an esthetic disorder rather than a disease. ${ }^{4}$ Treatment of such cases usually involves traditional, chemical, and cryosurgery. ${ }^{4-6}$ Surgery with gingivectomy normally reduced requiring local anesthesia, incision, and postoperative management with periodontal dressing. ${ }^{7}$ On the contrary, chemical surgery with phenol may have a toxic side effect. ${ }^{8}$ Therefore, many people are hoping for a simple alternative technique instead of these surgical techniques in the treatment for this disorder.

Nd:YAG and argon lasers are reported to be useful in removing gingival pigmentation due to their deep tissue penetrability and selective destruction of pigmented cells found in the basal cell layer under epithelium. ${ }^{9,10}$ Color dependency and tissue penetration are thought to be necessary for treatment of melanin pigmentation. However, it has been reported that depth of thermal damage to argon and Nd:YAG laser extend up to 200 and $600 \mu \mathrm{m}$,respectively. ${ }^{11}$ Therefore, such penetrability may damage the underlying alveolar bone covered by thinner oral mucosa.

The low power is considered beneficial for the treatments of hypersensitive dentin, herpes labialis, and oral ulcération. ${ }^{7-10}$ Its effect is reported to be due not to a heating process, but to radiation. ${ }^{12-14}$ Recently, a middlepower semiconductor laser by which soft tissue can be cut has been developed and is already being used in dentistry as well as in medicine. Semiconductor diode (SCD) laser is another popular and available device in the clinical field. It has been used for removal of gingival hyperpigmentation.

\section{MATERIALS AND METHODS}

A total five patients with four females and one male, aged 19 to 40 years participated in this clinical study. Four patients were nonsmokers and one was a smoker. They presented the gingival melanin hyperpigmentation at the anterior part of the upper and/or lower gingiva (Fig. 1). All patients were periodontally healthy and had no systemic diseases. Informed consent had been obtained from each patient prior to treatment. 


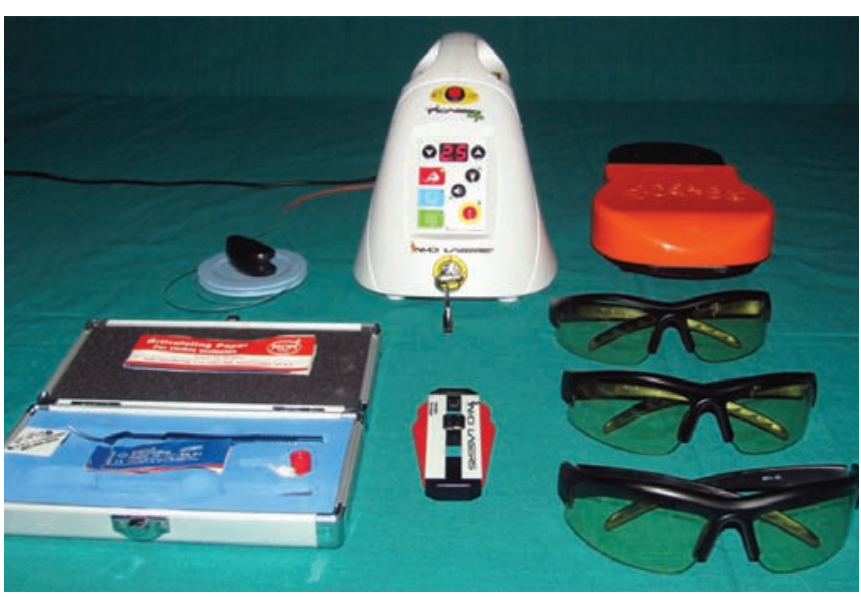

Fig. 1: Semiconductor diode laser used in study

\section{Laser Device}

Semiconductor diode laser (Fig. 1) used in this study with continuous wavelength $810 \mathrm{~nm}$ was applied at $3 \mathrm{~W}$ of power via pencil sized hand piece containing a $300 \mu \mathrm{m}$ lasing fiber was used to gently remove the gingival epithelium along with a layer of underlying connective tissue. The procedure was performed in a contact mode. The remnants of the charred ablated tissue were removed using a sterile dampened gauze soaked in saline. Periodontal dressing was placed on the operated site. Analgesics were prescribed. No antibiotics were given. Patients were asked to avoid trauma to the treated gingiva and to refrain from acidic and hot food for 1 week. Chlorhexidine mouthwash $(0.12 \%)$ for 2 weeks was prescribed. Safety glasses of specific wavelength as provided by the manufacturer were worn by the operator, patient, and assistant. Highly reflective instruments or instruments with mirrored surfaces were avoided, as there could have been reflection of the laser beam. Furthermore, care was taken to avoid laser in the presence of explosives and inflammable material.

\section{Clinical Evaluation}

Clinical parameters, such as wound healing and pain and were evaluated immediately after and then at 1 week, 1 month, and 3 months of surgery. Color of pigmentation was evaluated after 1, 3, 6, and 12 months postoperatively. Histological examination was performed preoperatively and 12 months postoperatively.

Preoperative (Fig. 2) and postoperative (Fig. 3) observations about the gingival melanin pigmentation were made according to Dummett-Gupta Oral Pigmentation Index scoring criteria given by Dummett and Gupta ${ }^{15}$ : 0 - No clinical pigmentation (pink gingiva)

1 - Mild clinical pigmentation (mild light brown color)

2 - Moderate clinical pigmentation (medium brown or mixed pink and brown color)

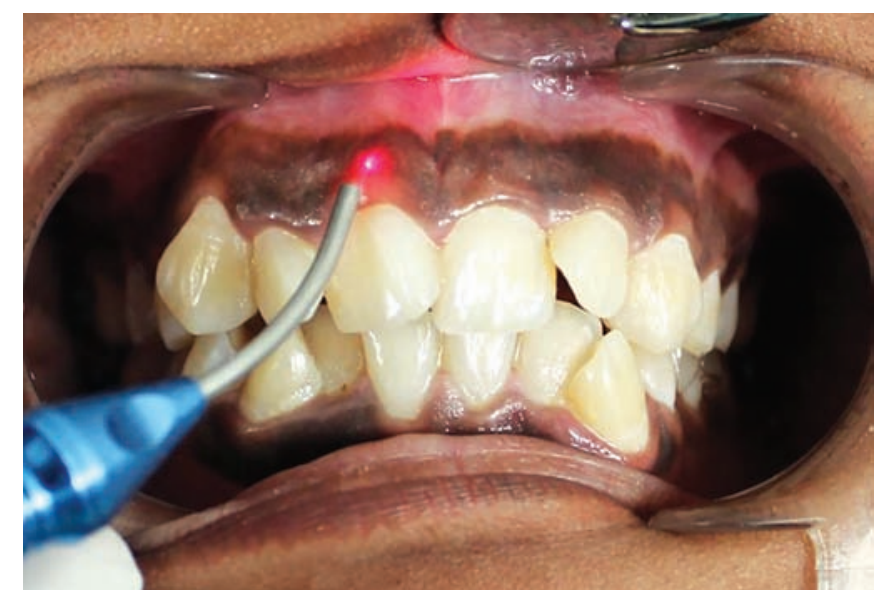

Fig. 2: Preoperative picture of patient

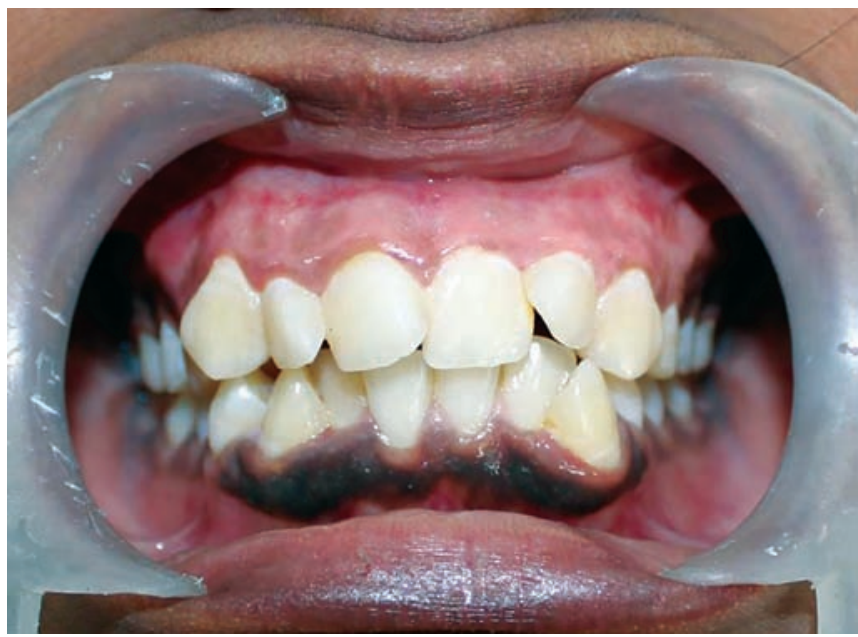

Fig. 3: Postoperative picture of patient

3 - Heavy clinical pigmentation (deep brown or bluish black color)

Wound healing was observed: (a) Complete epithelization, (b) incomplete epithelization/partial epithelization, (c) ulcer, and (d) tissue defect. Pain was evaluated by VAS score. If the score was 0 , then no pain; and scores between 0.1 and 3.0 were recorded as slight pain; scores between 3.1 to 6.0 was considered as moderate pain; and scores between 6.1 to 10 were recorded as severe pain. ${ }^{16}$ Histological examination was performed before (Fig. 4) and 1 year after treatment (Fig. 5). Biopsies were taken from unlashed and lashed areas of the gingiva. Sample were then fixed with buffered formalin solution and embedded in paraffin. Histological sections were examined using Fontona-Masson staining.

\section{RESULTS}

During the procedure, laser ablated the gingival epithelial surface little by little to reach the pigment without causing any bleeding which was beneficial for clear visualization. Removing deeper pigment resided below basal cell layer caused some bleeding spots which were stopped 


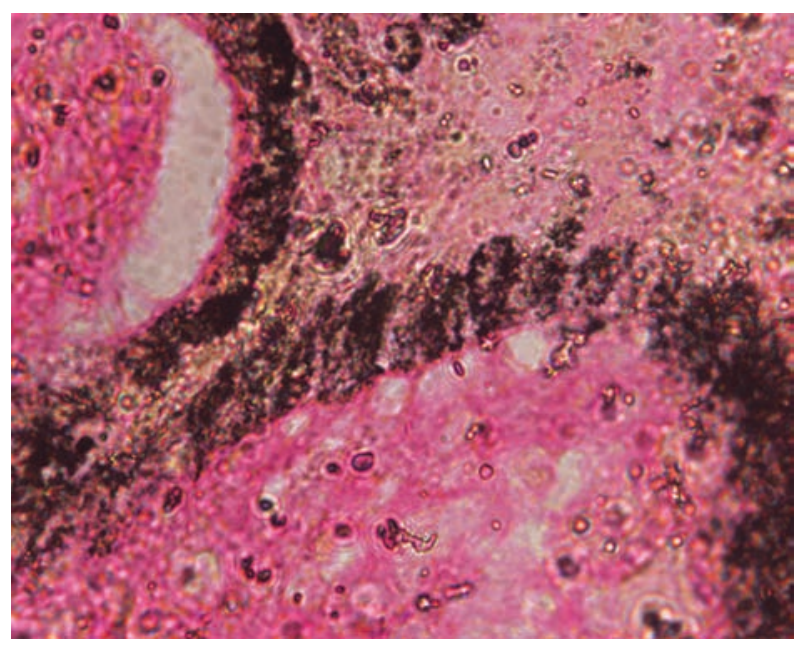

Fig. 4: Preoperative picture after Fontana-Masson staining taken at $100 \times$ resolution

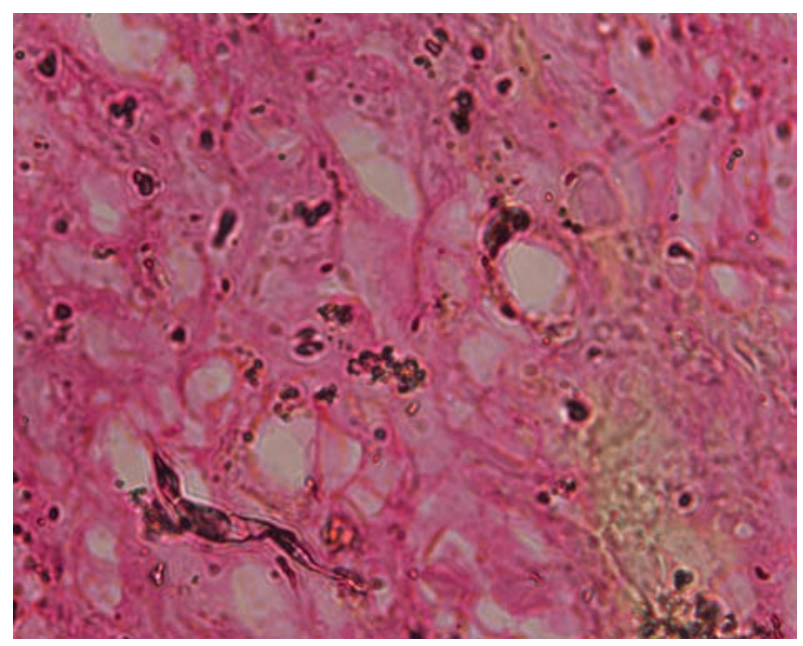

Fig. 5: Postoperative picture after Fontana-Masson staining taken at $100 \times$ resolution after 1 year by laser coagulation mode. Laser ablation of pigmented epithelium immediately produced a melanin pigmentfree surface without any carbonization. The lased wound looked fresh with no bleeding. Healing was good in 1 week with pink color comparable with nontreated areas that is bluish black in color, resulting in a singinificant improvement in esthetic appearance. Complete epithelization takes place after 2 weeks. There was slight pain observed after 24 hours in two cases and slight burning sensation during laser irradiation in two cases. The color of gingiva was pink. The color was observed 1, 6, and 12 months following the completion of therapy. All patients showed satisfactory results throughout the entire examination period.

\section{Histological Examination}

Before irradiation, the pigmentation consisted of melanin granules in the basal cell layer with Fontona-Masson staining. After 1 year, no melanin granules were observed in three cases, but slight melanin granules were observed in two cases.

\section{DISCUSSION}

Gingival hyperpigmentation is caused by excessive deposition of melanin located in the basal and suprabasal cell layers of the epithelium. Various depigmentation techniques have been employed using both nonsurgical and surgical procedures, such as scalpel surgery; free gingival autografting; cryosurgery; electrosurgery; chemical agents, such as 90\% phenol and 95\% alcohol; abrasion with diamond burs; and lasers like Nd:YAG laser, diode laser, and $\mathrm{CO}_{2}$ laser. Lasers are new in the field of dentistry and have been used in the field of periodontics for removal of calculus, soft tissue excisions, depigmentation, curettage, mucogingival surgeries like frenectomies, operculum removal, coagulation of graft donor site, and exposure of soft tissue covering osseointegrated implants. Of the most widely used soft tissue lasers are the diode lasers which are manufactured from semiconductor crystals using some combination of aluminum or indium, gallium, and arsenic. The laser is used for depigmentation of the gingiva because the wavelength of the laser is highly absorbed by the pigmented tissue containing hemoglobin, melanin, and collagen chromophores and little absorbance by the hard dental tissues. Therefore, this wavelength is safe and well indicated for (1) soft oral tissue surgeries in regions near dental structures, (2) cutting, (3) vaporization, (4) curettage, (5) blood coagulation, and (6) hemostasis. The available wavelengths of dental use range from $800 \mathrm{~nm}$ for the active medium containing aluminum to $900 \mathrm{~nm}$ for the active medium composed of indium. ${ }^{17}$

In this study, the SCD laser proved to be effective for removal of melanin pigmentation. All patients showed a normal appearance after following treatment. Difficulty was incurred while removing melanin in the region of the gingival papilla. This may be due high activity of pigmented cells in the area and the shorter treatment time for full depigmentation in SCD laser. Pain reduction after laser application may be attributed to the protein coagulum formed on the wound surface acting as biologic dressing.

According to Azzeh, ${ }^{18}$ the advantages of laser use are a relatively bloodless postsurgical course, sterilization of wound site, minimal swelling and scarring, little mechanical trauma reduction of surgical time, and high patient acceptance. However, Lagdive et $\mathrm{al}^{17}$ stated that to obtain the desired success of periodontal treatment without damage to surrounding tissues, the appropriate laser parameters, such as power energy, energy density, and time of irradiation have to be used. Therefore, in the present study, throughout the depigmentation procedure, 
the laser was cautiously used to avoid injury to the tooth surface and adjacent tissues. In addition, the thin gingival tissue around the root prominence was ablated gently. ${ }^{19}$

\section{Repigmentation}

In the present study, repigmentation was only observed in two out of five cases. Hirschfeld and Hirschfeld ${ }^{5}$ used $90 \%$ phenol and 95\% alcohol to remove areas of hyperpigmentation in 20 patients. Repigmentation soon developed in three patients, and the rest of the patients had the same results within a short period. Hu et $\mathrm{al}^{20}$ favored the "migration theory" for the mechanism of repigmentation. Active melanocytes from the normal skin and hair matrix proliferate and migrate into depigmented areas. Dummett ${ }^{21}$ defined oral repigmentation as the clinical reappearance of melanin pigment following a period of clinical depigmentation of the oral mucosa due to chemical, thermal, surgical, pharmacologic, or idiopathic factors. Tal and Stahl ${ }^{22}$ removed pigmented keratinized gingiva in two Jewish Yemanite adult males, who had moderate or heavily pigmented gingiva. After surgery, the exposed lamina propria was covered by the periodontal pack for 7 to 10 days. Healing was uneventful and surgically treated areas in both patients remained depigmented over the first 2 years. After 32 months, some pigmentation was found in one of the patients, and with the exception of two limited sites, the areas were completely pigmented after 7 years. The surgically treated area in the second patient remained depigmented over 8-year follow-up period. Bergamaschi et $\mathrm{al}^{23}$ reported a complete recurrence of gingival pigments after 3 years of follow-up after a gingivectomy procedure. Tamizi and Taheri ${ }^{24}$ reported repigmentation 1 year after using a Free gingival autograft for depigmentation. Nakamura et $\mathrm{al}^{25}$ reported that despite the lack of recurrence during the 1st year of follow-up, there was repigmentation in four of the seven cases treated by $\mathrm{CO}_{2}$ laser, almost equal to the postoperative state, at 24 months. Esen et $\mathrm{al}^{26}$ reported repigmentation in 2 cases out of 10 during 24-month follow-up after using the super pulsed mode of a $\mathrm{CO}_{2}$ laser.

In present study, repigmentation was observed only in one smoker patient after 12 months. Smoking activates the melanin production as well. However, severity of pigmentation was less than before treatment. The exact mechanism of repigmentation is not known, but according to migration theory, active melanocytes from adjacent pigmented tissue that migrated to treated area cause failure. ${ }^{27}$

\section{CONCLUSION}

This clinical and histological study indicates that SCD laser can be easily and effectively used for removal gin- gival hyperpigmentation. However, esthetic outcome may not last in the long run.

\section{CLINICAL SIGNIFICANCE}

Semiconductor diode laser can be easily and effectively used for removal gingiva hyperpigmentation. Ablation of gingival hyperpigmented areas were accomplished without any bleeding complication or slight pain or no pain observed, provided clean field during time of procedures. There was uneventful healing without any complication, and no recurrence or slight recurrence of pigmentation had been found in 12 months follow-up.

\section{ACKNOWLEDGMENT}

Head and the Chandra Dental College and Hospital, Barabanki, Uttar Pradesh, India, is acknowledged for providing all necessary facilities required for this study.

\section{REFERENCES}

1. Tong AK, Tan OT, Boll J, Parrish JA, Murpy GF. Ultrastructure: effects of melanin pigment on target specificity using pulsed dye laser (577 nm). J Invest Dermatol 1987 Jun;88(6):747-752.

2. Aderson RR, Margolis RJ, Watanabe S, Flotte T, Hruza GJ, Dover JS. Selective photothermolysis of cutaneous pigmentation by Q-Switched Nd:YAG laser pulses at 1064, 532 and 355 nm. J Invest Dermatol 1989 Jul;93(1):28-32.

3. Oshiro T. Laser treatment for Naevi. In: laser treatment for Naevi. England: West Sussex; 1995. p. 121-232.

4. Glickman I, Smulow JB. Gingiva pigmentation In: periodontal disease. Philadelphia (PA): WB Saunders; 1974. p. 4.

5. Hirschfeld I, Hirschfeld L. Oral pigmentation and method of removing it. Oral Surg Oral Med Oral Pathol 1951 Aug;4(8):1012-1016.

6. Tal H, Landsberg J, Kozlovsky A. Cryosurgical depigmentation of the gingiva - a case report. J Clin Periodontol 1987 Nov;14(10):614-617.

7. Kinoshita S, Wen R. A color atlas of periodontics. St. Louis, Tokyo: St Louis Mosby.Yearbook; 1985. p. 190-196.

8. Ayress S. Dermal changes following application of chemical cauterants to aging skin. Arch Dermatol 1960 Oct;82:578-585.

9. Matsumoto K, Ochi K, Tachibana H, Wakabayashi H. Study on the removal of melanin pigmentation by Nd:YAG laser. Jap J Conserv Dent (in Japanese) 1986;29:1543-1547.

10. Trelles MA, Verkruysse W, Segui JM, Udaeta A. Treatment of melanotic spot in the gingiva by argon laser. J Oral Maxillofac Surg 1993 Jul;51(7):759-761.

11. Absten GT, Joffe SN. Laser-tissue interaction. In: lasers in medicines. 2nd ed. London: Chapman and Hall; 1989. p. 16-21.

12. Smith PW. The soft laser: therapeutic tool and popular placebo? Oral Surg Oral Med Oral Pathol 1988 Dec;66(6):654658.

13. Basford JR. Low-energy laser therapy: controversies and new research findings. Lasers Surg Med 1989;9(1):1-5.

14. Strang R, Moseley H, Carmichael A. Soft lasers - have they a place in dentistry? Br Dent J 1988 Sep 24;165(6):221-225.

15. Dummett CO, Gupta OP. Estimating the epidemiology of the oral pigmentation. J Natl Med Assoc1964 Sep;56:419-420. 
16. Rosa DA, Aranha AC, Eduardo CP, Akira A. Esthetic treatment of gingival melanin hyperpigmentation with Er:YAG laser: short term clinical observations and patient follow-up. J Periodontol 2007 Oct;78(10):2018-2025.

17. Lagdive S, Doshi Y, Marawar PP. Management of gingival hyperpigmentation using surgical blade and diode laser therapy: a comparative study. J Oral Laser Applications 2009;9:41-47.

18. Azzeh MM. Treatment of gingival hyperpigmentation by erbium doped: yttrium, aluminum, and garnet laser for aesthetic purpose. J Periodontol 2007 Jan;78(1):177-184.

19. Ko HJ, Park JW, Suh JY, Lee JM. Esthetic treatment of gingival melanin hyperpigmentation with a Nd:YAG Laser and high speed rotary instrument: comparative case report. J Periodontal Implant Sci 2010 Aug;40(4):201-205.

20. Hu F, Fosnaugh RP, Leney PF. In vitro studies on vitiligo. J Invest Dermatol 1959 Nov;33:267-280.

21. Dummett CO. Oral pigmentation: first symposium on oral pigmentation. J Periodontal 1960 Oct;31:356-360.
22. Tal H, Stahl SS. Healing following devitalization of sites within the periodontal ligament by ultralow temperatures. J Periodontol 1986 Dec;57(12):735-741.

23. Bergamaschi O, Kon S, Doine AI, Ruben MP. Melanin repigmentation after gingivectomy: a 5-year clinical and transmission electron microscopic study in humans. Int J Periodont Res Dent 1993;13(1):85-92.

24. Tamizi M, Taheri M. Treatment of severe physiologic gingival pigmentation with free gingival autograft. Quintessence Int1996 Aug;27(8):555-558.

25. Nakamura Y, Hossain M, Hirayama K, Matsumoto K. A clinical study on the removal of gingival melanin pigmentation with the $\mathrm{CO}_{2}$ laser. Lasers Surg Med 1999;25(2):140-147.

26. Esen E, Haytac MC, Oz IA, Erdogan O, Karsli ED. Gingival melanin pigmentation and its treatment with the $\mathrm{CO}_{2}$ laser. Oral Surg Oral Med Oral Pathol Oral Radiol Endod 2004 Nov;98(5):522-527.

27. Perlmutter $\mathrm{S}, \mathrm{Tal} \mathrm{H}$. Repigmentation of the gingiva following surgical injury. J Periodontol 1986 Jan;57(1):48-50. 